DOI: https://doi.org/10.3126/njdrs.v17i0.34949

\title{
Prominence of Improved Cook Stove (ICS) in Rural Area: End- Users Perspective
}

\author{
Gopal Khadka \\ Lecturer at Patan Multiple Campus \\ Department of Rural Development, Tribhuvan University, Nepal \\ Email for correspondence:khadkajigopal@gmail.com
}

\begin{abstract}
Improved cook stove (ICS) indicates improved form of traditional cook stove. It is more effective, efficient and safer to use. ICS is popular in rural villages of Nepal as a new innovation of clean cooking mechanism. To explore the end users views about the prominence of ICS in rural settings of urban areas, this study is conducted as the title of prominence of improved cook stove in rural areas: end-users perspectives. It is based on the theoretical assumption of cultural feminism. It employs exploratory research design and is based on both primary and secondary sources of data. The study revealed that there is high prominence of ICS in rural farm based society in Nepal. Women are endusers of ICS. Through women perspectives, health and sanitation; easy operation; time and resource saver; low maintenance are its major prominence. ICS helps to reduce the untiring busy schedule of women in household activities. It suggests that concerned authorities should make effective institutional frameworks to empower women in their homeland by optimum utilization of leisure time made possible by the use of ICS.
\end{abstract}

Key words: Improved cook stove, end-user, rural, gender equality, prominence, cultural feminism

\section{Introduction}

The term 'improved cook stove' refers broadly to any stove disseminated by governments and independently by NGOs, the private sector, and other organizations that significantly improves energy efficiency, reduces indoor pollution, and increases ease of use. For people in developed countries, burning fuel wood in an open hearth evokes nostalgia and romance. But in developing countries, the harsh reality is that several billion people, mainly women and children, face long hours collecting fuel wood, which is burned inefficiently in traditional biomass stoves. The smoke emitted into their homes exposes them to pollution levels 10-20 times higher than the maximum standards considered safe in developed countries. And the problem is not out of the ordinary. The majority of people in developing countries at present cannot afford the transition to modern fuels. Today, close to one half of the world's people still depend on biomass energy to meet their cooking and heating needs (Douglaset. al, 2012). Biomass especially fuel wood is a viable source of energy for cooking in Nepal. Fuel wood is considered as basic need of Nepalese people. ICS is a boon for farmers due to its significance uses in their livelihood. On one hand, it reduces the deforestation rate and on the other hand; it increases the status of health and sanitation of its users. ICS has no bias in terms of caste, class, gender, religion 
and ethnicity.

Improved cook stoves offer the world's poor many potential benefits leading to cleaner hearths and healthier lives. Compared to traditional stove models, ICS can be engineered to provide superior performance, burning biomass energy more cleanly and efficiently. As a result, indoor pollution from cooking smoke will be reduced, helping to mitigate respiratory and other diseases. In addition, less time will be required for cooking meals and collecting biomass fuels, allowing women and other family members more time for educational and other productive activities. All of these factors combined contribute indirectly to a reduction in poverty (Douglas et al. 2011; Saghir 2005; World Bank 1999). The common conclusion of various reports related to energy stated that clean cook stoves and fuels have the potential to reduce deaths from Smoke-related illnesses, mitigate climate change and lower air pollution. They can provide new sources of livelihoods for women and reduce the risk and drudgery of fuel collection and lower household expenditures on cooking fuel.

Women, who have responsibility to manage domestic burden related to the kitchen, are the end- users of ICS in Nepal. Villagers confirmed that ICS is their true friend due to its help to save time, to reduce work-load, to maintain health, and to make a job convenient. The gender issue assigns tightly defined roles to men and women in family and society, which creates discriminatory attitudes and practices throughout the society, have a foundation in the family setting and reflected in the regular household activities. Low access to family property, lack of education, low economic status, lack of access to social issues, regular domestic burden hide their decision making power. In Nepalese family system, women have low status. Prior studies about ICS focus the view of the family head i.e. male, which hides the reality so it is essential to explore the real view of the true user of actual technology for assessing its effectiveness. This study provides the real reflection of targeted consumers for concerned authority.

Things have changed for the better, but not for all women and not in all domains of gender equality. Progress has been slow and limited for some women in very poor countries, for those who are poor, even amid greater wealth, and for those who face other forms of exclusion because of their caste, disability, location, ethnicity, or sexual orientation (World Bank, 2011). Development of Technology and gender gap are inversely proportional with one another. Various researches prove that Modern technology helps to bridge the gender gap. This study is helpful for gender analysts to calculate the role of ICS technology for reducing gender gap in rural farmland.

ICS was introduced in Nepal in 1950s and continues to be relevant in the present context. AEPC/NRREP, together with other government, non-government and private organizations, is involved in developing and promoting different types of ICS in Nepal and so far more than 700,000 improved cook stoves have been installed in 63 districts (AEPC, 2005). ICS is economically and environmentally compatible to use. Being the successful Public Private Partnership (PPP) model, it earns millions of dollars every year through the carbon trading system. So, without any doubt it can be helpful to put a landmark contribution by increasing awareness and fostering the positive attitude among the Nepalese society towards the development of ICS to promote a clean development mechanism in Nepal

Women perform multiple roles and duties in society. Women are farmers and foodproviders; they are business people and traders; they are heads of households; they are mothers, careers and support workers; and they are community leaders, activists and rolemodels. Development affects men 
and women differently, often with a more negative impact on women. This can undermine women's role, status and position within society and therefore perpetuates their inequality (Regan, 2012). Women and men play different defined roles in energy production, distribution and utilization in households, communities and the market. Climate stresses on biomass resources are increasing the burden on many women, forcing them to travel even longer distances and spend more time fetching fuel wood, water and fodder. ICS services can improve women's socioeconomic status by reducing the time and effort involved in household chores and alleviating the health risk associated with current cooking practices. There are several small scale clean technologies that can improve safety, employment and entrepreneurial opportunities for women. Technologies reduce women's challenges and increase their independence. Majority of the Nepalese populations still use traditional cook stove and fuels such as fire wood, agricultural residues and dung cake etc inside their homes, which creates various socioeconomic, environmental and health related adverse impacts on human health especially women. ICS has played a vital role in improving the health status and thus uplifting the living standard of rural people by providing clean fuel.

\section{Theoretical Modality}

This study is based on the key assumptions of cultural feminism modality to analyze the prominence of ICS in rural areas. It shows that there is 'female nature' of 'female essence'. It assumes that due to the cultural attributes, there are different perspectives between male and female towards the same phenomena, event or object. So the perspective of men and women are varying about the prominence of ICS. Cultural feminism is a variety of feminism which emphasizes essential differences between men and women, based on biological differences in reproductive capacity. Cultural feminism attributes to those differences distinctive and superior virtues in women. What women share, in this perspective, provides a basis for "sisterhood," or unity, solidarity and shared identity? Thus, cultural feminism also encourages building shared women's culture. The phrase "essential differences" refers to the belief that gender differences are part of the essence of females or males, that the differences are not chosen but are part of the nature of woman or man. Cultural feminists differ as to whether these differences are based on biology or enculturation. Those who believe differences are not genetic or biological, but are cultural; conclude that women's "essential" qualities are so ingrained by culture that they are persistent (Lewis, 2019).

\section{Objective}

- To explain the prominence of improved cook stove in rural area through end-users perspective.

\section{Methods and Materials}

This study employs the exploratory research design to explore the prominence of biogas in rural settings of urban areas through end-users perspective. It is based on the idea of cultural feminism. Ratuwa Mai municipality ward no 10, Morang district is a study area where 180 households have improved cook stoves. 100 women using ICS in their house since one year were selected as respondents through purposive sampling procedure for detailed study of ICS technology in its natural real-life context. It is based on both primary and secondary data. The primary data were collected through in-depth face-to-face structured interviews and observations. The secondary data were collected by reviewing ICS-related literature through search engines. After Pretesting the question of in-depth 
structured interview, Data were collected with minute observation by the active initiation of the researcher himself. The collected data has been checked, coded, categorized, organized and converted into a master data sheet. Data was presented in Table and figure by using ms-word and ms-excel. Data is analyzed descriptively through percentage, frequencies and average mean value.

Result and Discussion: Socio Demography of the Respondents

Table 1. Social Demographic Characteristics of the Respondents

\begin{tabular}{lcc}
\hline Variables & Frequency & Percentage \\
\hline 1. Ethnicity: Brahmin & 25 & $25 \%$ \\
Chhetri & 35 & $33.3 \%$ \\
Janajati & 40 & $41.7 \%$ \\
Total & $\mathbf{1 0 0}$ & $\mathbf{1 0 0 \%}$ \\
2. Age groups (in years): 20-30 & 22 & $22 \%$ \\
30-40 & 35 & $35 \%$ \\
40-50 & 43 & $43 \%$ \\
Total & $\mathbf{1 0 0}$ & $\mathbf{1 0 0 \%}$ \\
3. Occupation: Farming & 85 & $85 \%$ \\
Service & 15 & $15 \%$ \\
Total & $\mathbf{1 0 0}$ & $\mathbf{1 0 0 \%}$ \\
4. Education: Illiterate & 10 & $10 \%$ \\
Literate & 90 & $90 \%$ \\
Total & $\mathbf{1 0 0}$ & $\mathbf{1 0 0 \%}$ \\
5. Level of education: Primary & 35 & $38.8 \%$ \\
Secondary & 40 & $44.4 \%$ \\
Higher secondary & 15 & $16.8 \%$ \\
Total & 90 & $100 \%$ \\
6. Religion: Hindu & 55 & $55 \%$ \\
Buddhist & 20 & $20 \%$ \\
Kirat & 25 & $25 \%$ \\
Total & $\mathbf{1 0 0}$ & $\mathbf{1 0 0 \%}$ \\
7. Family size (person): 1-4 & 35 & $35 \%$ \\
5-8 & 65 & $65 \%$ \\
Total & $\mathbf{1 0 0}$ & $\mathbf{1 0 0 \%}$ \\
8. Number of children (person):1-2 & $35 \%$ \\
3-4 & $\mathbf{1 0 0}$ & $\mathbf{1 0 0 \%}$ \\
Total & & $\mathbf{1 0 0}$ \\
\hline
\end{tabular}

(Field Survey, 2020)

On the basis of table 1, respondents have heterogeneous nature in terms of age, ethnicity, religion and occupation. No one ethnic group is in majority. Janjati group includes Tamang, Rai and Limbu. They belong to 20-50 yrs age groups. Most of them follow farming as their occupation and Hinduism as their religion. Most of them (90\%) are literate. Large number of literate respondents has completed secondary level education. They have up to 8 members in the family and up to 4 numbers of children. All of them resided in small size family. 15 respondents engaged as employed in primary 
level boarding school and cooperative institutions.

\section{Prominence of ICS in terms of Health and Sanitation}

Table 2 reveals the prominence of ICS in terms of health and sanitation. The responses of the respondents on 19 study variables based on five rating scales* ( $1=$ Strongly Agree, $2=$ Agree, $3=$ Neutral, $4=$ Disagree, $5=$ Strongly Disagree) found that ICS helps to keep clean and hygienic surrounding of home; convenience to manage kitchen gardens and production of organic food stuff .Majority of respondents agreed that due to the absence of a smoky environment, the interior of the kitchen is clean; easy and fast to wash the utensils; long life of utensils and reduction of hand skin problems .These all are related to smoke and dust of firewood.

Table 2. Prominence of Biogas in Terms of Health and Sanitation

\begin{tabular}{|c|c|c|c|c|c|c|c|}
\hline \multirow[t]{2}{*}{ S. N. } & \multirow[t]{2}{*}{ Variables } & \multicolumn{6}{|c|}{ Five Rating Scales* } \\
\hline & & 1 & 2 & 3 & 4 & 5 & Total \\
\hline 1 & Clean and hygienic surrounding of home & $25 \%$ & $65 \%$ & $8 \%$ & $1 \%$ & $1 \%$ & $100 \%$ \\
\hline 2 & Clean and hygienic interior of kitchen & $20 \%$ & $66 \%$ & $11 \%$ & $2 \%$ & $1 \%$ & $100 \%$ \\
\hline 3 & Easy to manage kitchen garden & $8 \%$ & $74 \%$ & $10 \%$ & $3 \%$ & $5 \%$ & $100 \%$ \\
\hline 4 & Reduction of eye diseases & $16 \%$ & $75 \%$ & $7 \%$ & $1 \%$ & $1 \%$ & $100 \%$ \\
\hline 5 & Reduction of respiratory diseases & $12 \%$ & $78 \%$ & $7 \%$ & $2 \%$ & $1 \%$ & $100 \%$ \\
\hline 6 & Reduction of skin problems & $14 \%$ & $75 \%$ & $5 \%$ & $4 \%$ & $2 \%$ & $100 \%$ \\
\hline 7 & Safe to operate than traditional cook stove & $16 \%$ & $70 \%$ & $8 \%$ & $5 \%$ & $1 \%$ & $100 \%$ \\
\hline 8 & Easy and fast to clean utensil & $25 \%$ & $70 \%$ & $3 \%$ & $2 \%$ & - & $100 \%$ \\
\hline 9 & Long life of utensil & $20 \%$ & $67 \%$ & $10 \%$ & $3 \%$ & - & $100 \%$ \\
\hline 10 & No smell of smoke in food & $18 \%$ & $66 \%$ & $12 \%$ & $3 \%$ & $1 \%$ & $100 \%$ \\
\hline 11 & Sanitation in overall locality & $18 \%$ & $60 \%$ & $13 \%$ & $6 \%$ & $3 \%$ & $100 \%$ \\
\hline 12 & Tasty food than traditional cook stove. & $70 \%$ & $15 \%$ & $10 \%$ & $3 \%$ & $2 \%$ & $100 \%$ \\
\hline 13 & Decrease fuel wood consumption & $80 \%$ & $15 \%$ & $5 \%$ & - & - & $100 \%$ \\
\hline 14 & Food remain warm for long duration & $75 \%$ & $15 \%$ & $5 \%$ & $5 \%$ & - & $100 \%$ \\
\hline 15 & Easy to cook food & $65 \%$ & $15 \%$ & $15 \%$ & $3 \%$ & $2 \%$ & $100 \%$ \\
\hline 16 & Decrease indoor air pollution & $70 \%$ & $20 \%$ & $10 \%$ & - & - & $100 \%$ \\
\hline 17 & Reduce fire hazards in kitchen. & $19 \%$ & $55 \%$ & $16 \%$ & $4 \%$ & $6 \%$ & $100 \%$ \\
\hline 18 & Reduce drudgery of women & $13 \%$ & $65 \%$ & $12 \%$ & $6 \%$ & $4 \%$ & $100 \%$ \\
\hline 19 & Improve health status of women. & $25 \%$ & $69 \%$ & $6 \%$ & - & - & $100 \%$ \\
\hline
\end{tabular}

(Field Survey, 2020)

Majority of respondents agreed that ICS improve health status of women. Creation of a clean and healthy environment reduces the intensity of respiratory, skin and air borne diseases. Majority of them agreed that ICS is easy and safe to use, the intensity of fire on people and houses is reduced after the use of ICS. Housewives/women are the main beneficiaries of the ICS.

\section{Social Prominence of ICS}

Table 3 reveals the response of the respondents about the prominence of ICS related to social aspects. The respondents views on 8 study variables with five rating scales* ( $1=$ Strongly Agree, 2=Agree, 3=Neutral, 4=Disagree, 5=Strongly Disagree) found that the ICS reduced their work load and saved time. It has reduced the workload of women in household activities because they have spent less time on cooking, cleaning utensils and collecting firewood. Majority of them use their leisure time in social work, entertaining and informational programs on Radio and TV. Therefore, introduction of 
ICS technology has indirectly contributed to empower rural women as majority of them were regularly watching the TV, listening to the radio and reading the newspaper during the leisure time created by using ICS in the house.

Table 3. Social Prominence of Improved Cook Stove

\begin{tabular}{llllllll}
\hline S. N. & Variables & Five Rating Scales* & & \\
& & 1 & 2 & 3 & 4 & 5 & Total \\
1 & Increase leisure time & $22 \%$ & $67 \%$ & $7 \%$ & $2 \%$ & $2 \%$ & $100 \%$ \\
2 & Increase Social interaction & $20 \%$ & $70 \%$ & $6 \%$ & $3 \%$ & $1 \%$ & $100 \%$ \\
3 & Effective management of family & $30 \%$ & $57 \%$ & $7 \%$ & $4 \%$ & $2 \%$ & $100 \%$ \\
4 & Involve in family interaction & $31 \%$ & $60 \%$ & $7 \%$ & $2 \%$ & - & $100 \%$ \\
5 & Involve in social work & $14 \%$ & $56 \%$ & $15 \%$ & $10 \%$ & $5 \%$ & $100 \%$ \\
6 & Assist children to complete their homework & $25 \%$ & $56 \%$ & $10 \%$ & $5 \%$ & $3 . \%$ & $100 \%$ \\
7 & Getting more information & $25 \%$ & $51 \%$ & $16 \%$ & $5 \%$ & $3 \%$ & $100 \%$ \\
8 & Involve on entertaining program & $13 \%$ & $67 \%$ & $10 \%$ & $7 \%$ & $3 \%$ & $100 \%$ \\
\hline
\end{tabular}

(Field Survey, 2020)

The responses of the respondents based on $d$ that after the installation of ICS, frequency of social interaction increased. Women have sufficient time for socialization and participation in social activities, meetings. From participating in various activities they get confident and strongly express their feelings to others. Women's group also organizes different kinds of programs to enhance and develop new skills like tailoring, farming and woolen sewing and also get opportunity to socialize. A majority of woman responds that high frequency of family interaction helps to understand the problems of family and manage them on time. They have enough time to motivate their children about homework. They can spend more time with family members nowadays.

\section{Limitation of ICS}

Table 4 revealed some limitation of ICS perceived by its end-users. The responses of the respondents on 6 study variables with five rating scales* (1=Strongly Agree, 2=Agree, 3=Neutral, $4=$ Disagree, $5=$ Strongly Disagree) found that most of the respondents agreed that there is no direct monetary income of ICS. They reported that there is difficult to use agriculture residues. They suggested that using big pot on ICS becomes problematic. They cannot replace broken parts of stove themselves. They feel burden to clean the chimney.

Table 4. Limitation of Improved Cook Stove

\begin{tabular}{llllllll}
\hline S. N. & Variables & \multicolumn{7}{c}{ Five Rating Scales } & & & Total \\
& & 1 & & 3 & 4 & 5 & $100 \%$ \\
$\mathbf{1}$ & Difficult to use agriculture residues & $66 \%$ & $21 \%$ & $8 \%$ & $3 \%$ & $2 \%$ & 100 \\
$\mathbf{2}$ & Difficult to cook on big pot & $65 \%$ & $23 \%$ & $6 \%$ & $4 \%$ & $2 \%$ & $100 \%$ \\
$\mathbf{3}$ & Difficult to replace broken parts of stove. & $70 \%$ & $15 \%$ & $10 \%$ & $5 \%$ & - & $100 \%$ \\
$\mathbf{4}$ & Difficult to clean the chimney & $59 \%$ & $31 \%$ & $5 \%$ & $3 \%$ & $2 \%$ & $100 \%$ \\
$\mathbf{5}$ & Difficult to cook fast through big flame & $65 \%$ & $16 \%$ & $10 \%$ & $4 \%$ & $5 \%$ & $100 \%$ \\
$\mathbf{6}$ & No direct monetary income & $80 \%$ & $20 \%$ & - & - & - & $100 \%$ \\
\hline
\end{tabular}

(Field Survey, 2020) 


\section{Conclusion}

There is no direct and immediate economic return of improved cook stove. It creates indirect benefit to all family members. Due to the immediate and close touch of women with kitchen related activities, it provides more benefit towards women than other family members. By reducing the rush schedule of managing firewood, it creates favorable time for socialization through watching TV, listening to radio and interaction with villagers. By reducing the smoky environment in the kitchen, it maintains sanitation and reduces the intensity of diseases. By reducing expenditure on costly fuel such as LPG, kerosene, coal and more firewood etc for cooking, it helps to boost up saving economically and forest area environmentally. Status of health and sanitation of women were positively changed through the use of improved cook stove. It helps to increase the life expectancy of people by reducing the risk of various communicable and non communicable diseases. Since the use of improved cook stove in the house, the living standard of women is uplifted. Healthy and happy women can create a heavenly environment in the family. It is essential to motivate villagers to use the improved cook stove by educating them about its direct and indirect benefit. The visible positive impacts on the health and livelihood of rural families utilizing improved cook stove have led their neighbors to adopt this systems as well. Women ICS users' groups must be formed and launch women empowerment programs by focusing on their leisure time to provide training of income generating skills, packaging and marketing of organic farm products through organic farming system. Provision of soft loans by financial institutions should be managed with group collateral. It creates direct benefits to women through their group solidarity politically, socially and economically. Concerned authority must understand the enduser needs and concerns and design a product that meets the needs and addresses the concerns. So it is essential to assess the needs and conditions of the end-user and to determine the suitability of the available ICS technologies to fit them. In Nepalese context women are the actual end users of the ICS system.

\section{References}

Alternative Energy Promotion Centre (2005). Improved cooking stove. Blog Post. Retrieved from https://www.aepc.gov.np/improved-cooking-stove

Douglas, B. F., Khandker, S., \& Samad, H. (2011). 'Energy Poverty in Rural Bangladesh'. Energy Policy, 39(2), 894-904.

Douglas B. F., Kumar, P. \& Openshaw, K. (2012). Cleaner hearths, better homes. New stoves for India and the developing world.New Delhi: Oxford University Press. Retrieved from http:// cleancookstoves.org/resources_files/cleaner-hearths-better.pdf

Lewis, J. J. (2019). Cultural feminism. Retrieved from

Regan, C. (2012). Women and development. Development education. Retrieved from https:// developmenteducation.ie/feature/women-development/

Saghir, J. (2005). 'Energy and poverty: Myths, links and policy issues', Energy working notes, No. 4. Washington, DC: The World Bank.

World Bank (1999). Fuel for thought: Environmental strategy for the energy sector. Washington, D.C.: The World Bank.

World Bank (2011). World development report 2012: Gender and development. Washington DC: Author. 